Meta

Journal des traducteurs

Translators' Journal

\title{
Réflexions sémantiques sur l'article dans l'oeuvre lexicographique
}

\section{Maria Aparecida Barbosa}

Volume 41, numéro 2, juin 1996

Traduction et terminologie au Brésil

Translation and Terminology in Brazil

URI : https://id.erudit.org/iderudit/001969ar

DOI : https://doi.org/10.7202/001969ar

Aller au sommaire du numéro

Éditeur(s)

Les Presses de l'Université de Montréal

ISSN

0026-0452 (imprimé)

1492-1421 (numérique)

Découvrir la revue

Citer cet article

Barbosa, M. A. (1996). Réflexions sémantiques sur l'article dans l'oeuvre lexicographique. Meta, 41(2), 265-274. https://doi.org/10.7202/001969ar
Résumé de l'article

L'auteur étudie certains aspects fondamentaux dans l'élaboration de l'énoncé définitoire de l'œuvre léxico graphique. Elle se penche sur l'importance de l'articulation des relations de signification dans la description de l'unité lexicale, prises en compte dans l'élaboration de la macro- et de la micro-structure de l'œuvre lexicographique ; puis sur les questions touchant la caractérisation rigoureuse des relations de co-hyponymie et de parasynonymie, fondamentales dans la constitution du système de renvois des dictionnaires et des vocabulaires. 


\title{
RÉFLEXIONS SÉMANTIQUES SUR L'ARTICLE DANS L'CEUVRE LEXICOGRAPHIQUE
}

\author{
MARIA APARECIDA BARBOSA \\ Universidade de São Paulo, São Paulo, Brésil
}

\begin{abstract}
Résumé
L'auteur étudie certains aspects fondamentaux dans l'élaboration de l'énoncé définitoire de l'cuvre lexicographique. Elle se penche sur l'importance de l'articulation des relations de signification dans la description de l'unité lexicale, prises en compte dans l'élaboration de la macro- et de la micro-structure de l'œuvre lexicographique; puis sur les questions touchant la caractérisation rigoureuse des relations de co-hyponymie et de parasynonymie, fondamentales dans la constitution du système de renvois des dictionnaires et des vocabulaires.
\end{abstract}

\section{Abstract}

This paper examines certain fundamental issues dealing with lexicographical definitions. It first focuses on the importance of sense relations in describing lexical units in the macrostructure and microstructure of lexicographical works. Then, it discusses the need for a rigorous description of co-hyponymic and parasynonymic relations, the basic components of the cross-reference system of dictionaries and vocabularies.

L'analyse sémantique et sémantico-syntaxique de l'unité lexicale constitue l'un des principaux objectifs du projet lexicographique (Rey et Delesalle 1974: 4). Cette analyse se réalise par la définition des descripteurs, qui se présente ainsi comme une paraphrase synonymique du terme défini. Il s'établit de cette façon, entre définition et terme défini, une relation d'opposition transitive, dans laquelle l'ensemble-intersection sémique doit être aussi étendu que possible, et l'ensemble-différence sémique aussi réduit que possible, étant donné qu'une opposition d'identité est extrêmement difficile à obtenir.

Certes, la nature de l'œuvre lexicographique - dictionnaire de langue, dictionnaire bilingue, vocabulaire technico-scientifique/spécialisé, glossaire, thesaurus - détermine la modalité de la définition, en tant que constituant obligatoire de la microstructure de l'énoncé lexicographique, ou, en d'autres termes, typologie de l'œuvre lexicographique et typologie de la définition se situent dans une relation déterminant/déterminé. Son éventuelle désarticulation conduit à l'appauvrissement des qualités discursives de l'œuvre, réduisant par là même son efficacité en tant qu'instrument d'emmagasinage et de récupération de l'information.

Nous examinerons, ici, certains aspects que nous considérons indispensables à l'élaboration de l'énoncé définitionnel de l'œuvre lexicographique, en respectant la nature, la typologie et la fonction de celle-ci.

Dans cette perspective, les concepts et attributs résultants peuvent être jugés grâce aux critères de précision/imprécision, universalité/non-universalité, univocité/ambiguïté, pertinence/non-pertinence, uniformité/non-uniformité.

L'utilisation rigoureuse de ces critères exige donc que soient prises en compte, dans la structure de la définition, dans l'analyse sémique et lexico-sémantique qui l'orientent, les relations de signification comme la synonymie, la parasynonymie, l'hypéronymie, l'hyponymie, la co-hyponymie et la paronymie.

Meta, XLI, 2, 1996 
La recherche réalisée et ici présentée, ainsi que ses résultats, font partie d'une recherche plus vaste, qui s'insère dans la ligne de recherche de «Lexicologie et Lexicographie», sous la direction de l'auteur, dans le cadre des enseignements de Doctorat en linguistique de l'Université de São Paulo, depuis 1975, constituée de divers projets parmi lesquels se détache la problématique relative à la politique, à la typologie et à la technologie de production des vocabulaires technico-scientifiques et spécialisés.

Nous nous proposons, dans cette recherche plus large, d'aborder les questions relatives aux méthodes de définition lexicographiques, qui comprennent des normes variées et spécifiques de l'analyse lexico-sémantique, en fonction des divers types de dictionnaires, de la nature, de l'étendue, de la quantité d'information et de la fin à laquelle ils se destinent. Nous nous proposons encore d'observer et de décrire «le savoir social et humain» conféré aux vocables par les groupes sociolinguistiques et culturels et, par les différents domaines d'expérience, de construire des modèles qui permettent une meilleure compréhension de la «vérité» du discours lexicographique, ainsi que l'élaboration d'une méthodologie qui rende possible la compatibilisation de concepts, dans des domaines spécifiques, afin de produire des vocabulaires technico-scientifiques adaptés à la culture brésilienne, qui puissent contribuer au progrès de la science et de la technologie dans ce pays, l'une des conditions de la souveraineté nationale.

Nous nous sommes penchés, dans ce travail, d'une part, sur les aspects relatifs à l'importance de l'articulation de l'étude des types de relations de signification de l'unité lexicale - polysémie, homonymie, homosémie, hypéronymie, hyponymie, etc. — , pour la construction de la macro- et de la microstructure de l'œuvre lexicographique; d'autre part, sur les questions qui concernent le besoin d'une caractérisation rigoureuse des relations de co-hyponymie et de parasynonymie, fondamentales pour la constitution du système de renvois des dictionnaires et des vocabulaires mentionnés ci-dessus.

Appliquer les relations de signification à la macrostructure du dictionnaire consiste à établir le critère lexico-sémantique de décision sur les vocables (acceptions) qui doivent être regroupés dans le même article ou sous la même entrée, et sur ceux qui doivent être considérés non pas comme des vocables du même lexème mais comme des lexèmes distincts. En somme, de telles relations permettent de déterminer ce que l'on doit considérer comme un lexème et ce que l'on doit considérer comme un vocable (Muller 1968: 136). En d'autres termes, elles permettent de vérifier s'il s'agit d'un lexème et de ses vocables - avec des acceptions différentes mais non disjointes, un noyau sémique commun étant maintenu - , ou s'il s'agit de deux ou de plusieurs lexèmes. Dans le premier cas, nous avons un seul lexème polysémique, dans le second, des lexèmes homonymes. D'autres applications importantes sont possibles. De cette façon, si la macrostructure ou nomenclature de l'œuvre est constituée par l'ensemble des entrées, ordonnées, soumises à une lecture verticale, les notions de polysémie et d'homonymie, par exemple, peuvent changer substantiellement le nombre des entrées et l'organisation même des articles, ou entrées, sur le plan vertical, dans le cas, entre autres, d'une distribution des entrées par ordre alphabétique. Quant aux notions d'hypéronymie/hyponymie et de co-hyponymie, elles semblent composer un excellent modèle de relations de signification, pour l'organisation et la distribution des entrées par champs lexico-sémantiques.

Appliquer les relations de signification à la microstructure correspond à préciser les critères lexico-sémantiques et d'analyse sémique (sémantico-syntaxique) de la définition et de la compatibilisation des sèmes, de façon à représenter fidèlement le réseau lexico-sémantique inscrit dans le système, dans les normes et leurs relations, effectivement constatées dans l'usage social. La microstructure de base, sur laquelle nous nous sommes penché dans un travail précédent (Barbosa 1989: 65), est constituée par l'ensemble des «informations» ordonnées qui suivent l'entrée et ont une structure 
constante, correspondant à un programme et à un code d'informations, applicables à n'importe quelle entrée. Nous dénommons cet ensemble Entrée + Énoncé Lexicographique l' «article». L'«article» minimal, donc, possède deux constituants : entrée + définition. Toutefois, il faut observer que la définition et tous les autres constituants de l'énoncé lexicographique, commme, également, la méthodologie même qui oriente la construction de celui-ci, s'organisent en fonction de la nature de l'œuvre lexicographique dans laquelle ils s'intègrent. Ainsi, outre ce programme minimal de microstructure, il existe toute une gamme de possibilités informationnelles. En nous limitant au constituant définitionnel, nous vérifions que les relations de significations du type hypéronymie/hyponymie sont, au côté de la parasynonymie (acceptions), également importantes. En effet, elles doivent être prises en compte non seulement dans la présentation des «entrées» (macrostructure) et des sous-entrées (généralement utilisées dans la microstructure - cas de la chaîne syntagmatique, de la chaîne phonétique, etc.), mais également et en ce qui concerne la présentation des attributs, où il convient que le lexicographe soit attentif à la hiérarchie des hyperonymes/hyponymes, afin d'essayer de garantir la spécificité de la définition.

Appliquer les relations de signification au processus de renvois correspond à établir des critères selon lesquels les relations antérieurement citées doivent être explicitées, d'après des critères complémentaires, relatifs à la fréquence et à la distribution régulière entre les locuteurs de la langue (dictionnaire de langue) ou à l'intérieur d'un univers de discours. En ces termes, les notions de parasynonymie et de synonymie nous permettent de faire un renvoi à des lexèmes équivalents; les notions d'hypéronymie, d'hyponymie et de paronymie nous permettent les renvois du type voir, par exemple. La distinction précise et correcte entre parasynonymes et co-hyponymes nous empêche de faire des renvois inadéquats de mots «équivalents» entre co-hyponymes, puisque ce processus n'est recommandé que pour le traitement des synonymes et parasynonymes (Barbosa 1989: 325).

Arrivés à ce point, il convient de définir, ne serait-ce que d'une façon sommaire, la parasynonymie et la co-hyponymie, en détachant leurs traits communs et divergents. D'après Geckeler (1976: 285), les critères qui permettent d'affirmer si différents lexèmes ont le même signifié sont habituellement les suivants : a) capacité illimitée de substitution dans tous les contextes; b) coïncidence, dans le signifié dénotatif et dans le signifié connotatif.

Il semble, donc, que seuls de rares cas remplissent de telles conditions, par exemple, les formes lexicales techniques et leurs correspondants vulgaires, lorsqu'on les considère dans une perspective intra-univers de discours, ou les formes auxquelles on attribue une relation d'équivalence dans un contexte déterminé. $\mathrm{Si}$ les cas de synonymie sont très rares, il est bon de considérer, alors, les cas de parasynonymie, qui, d'après Galisson (1973 : 103), sont des termes qui peuvent être considérés comme ayant le même sens mais dont les distributions ne sont pas exactement équivalentes. Se caractérisent comme des cas de parasynonymie, entre autres, les paraphrases culturelles, les différentes «visions» pour le même schéma conceptuel, les variantes diachroniques, diatopiques, diastratiques et diaphasiques (Pottier 1968: 127; 1974:94); les paraphrases lexicales, les paraphrases déictiques, les paraphrases pragmatiques (Brekle 1974: 72).

Le même type de structure est sous-jacent à tous les cas de parasynonymie: ce sont des formes qui appartiennent au même microsystème sémantico-syntaxique, elles maintiennent entre elles une relation d'opposition transitive quant au signifié, c'est-à-dire un ensemble-intersection ample, avec un nombre de traits sémiques communs significatif, un ensemble-différence qui contient, au moins, un sème qui les distingue; ce sont des formes qui maintiennent une implication réciproque, bilatérale ou d'équivalence; des 
formes qui ont une équivalence du sens cognitif mais pas du sens affectif; qui, pour ces raisons, peuvent être substituées l'une à l'autre, selon le temps et le lieu, au niveau du système; de toute façon, la différence entre la synonymie/parasynonymie est une question de degré; d'après Lyons (1979: 476), tout ensemble d'unités lexicales peut être organisé selon une échelle de ressemblance et différence, de sorte que $\boldsymbol{a}$ et $\boldsymbol{b}$ puissent être considérés de sens identique (synonymes stricto sensu), $\boldsymbol{a}$ et $\boldsymbol{c}$ relativement semblables (synonymes lato sensu), $\boldsymbol{a}$ et $\boldsymbol{d}$ moins semblables et ainsi de suite. C'est pour cela que, par exemple, dans la traduction d'une langue à l'autre, il n'y a jamais de synonymes mais un degré plus ou moins grand dans l'«application» des mots.

Certaines structures sont communes à la parasynonymie et à la co-hyponymie, fait qui entraîne, parfois, une certaine confusion entre ces relations de signification; selon d'autres perspectives, elles ne peuvent absolument pas être considérées comme identiques. En effet, les co-hyponymes sont, par définition, des termes sémantiquement proches, puisqu'ils ont un hyperonyme commun; le degré de proximité entre ce type d'éléments lexicaux varie d'un microsystème à l'autre, autorisant une sous-classification des co-hyponymes en co-hyponymes proches et co-hyponymes distants (Galisson 1973: 103), d'où il résulte que les premiers sont plus facilement confondus avec les parasynonymes. Toutefois, un examen plus attentif de la co-hyponymie et de la parasynonymie montre que s'ils présentent certaines structures communes, ils en possèdent d'autres qui sont bien différentes, définissant ainsi une relation d'opposition transitive.

Dans l'ensemble intersection, sont communs les aspects suivants: a) les parasynonymes, tout comme les co-hyponymes, se rattachent à un terme supérieur surordonné (hypéronyme); b) par conséquent, dans les deux cas, il y a opposition transitive; c) les mots parasynonymiques et co-hyponymiques, quant au paradigme de la sémantique grammaticale, ont la même capacité à recevoir l'incidence de signes grammaticaux compatibles avec la classe grammaticale à laquelle ils appartiennent; d) quant aux paradigmes de la sémantique lexicale, ils présentent des différences de sèmes lexicaux dans la relation mot/mot; e) les paradigmes des relations syntagmatiques possèdent, quant à eux, les mêmes possibilités de combinatoire syntaxique.

Toutefois, dans l'ensemble-différence: a) le principal aspect distinctif entre les mots parasynonymes et les co-hyponymes réside dans le paradigme de référentialité, c'est-à-dire que les premiers ont la même référence cognitive et des références affectives distinctes, les seconds, des références cognitives et affectives distinctes; b) par conséquent, les premiers ont presque la même distribution, c'est-à-dire qu'ils peuvent être commutables dans de nombreux contextes sémantiques; les seconds ne sont pas commutables entre eux, du point de vue sémantique; c) l'ensemble-différence, dans la relation de parasynonymes, peut être constitué de peu de sèmes et même être neutralisé, selon le contexte, ou annulé avec le temps; bien que ceci puisse se vérifier également avec des co-hyponymes proches, cela se produit rarement avec des co-hyponymes distants, puisque leurs différences dénotatives et connotatives respectives sont clairement distinctes, et les structures de la sémantique lexicale, très diverses; d) la relation d'équivalence n'existe qu'entre les parasynonymes. Schématiquement, nous avons: 


\section{Parasynonymie}

Macroparadigme sémant.-syntax.

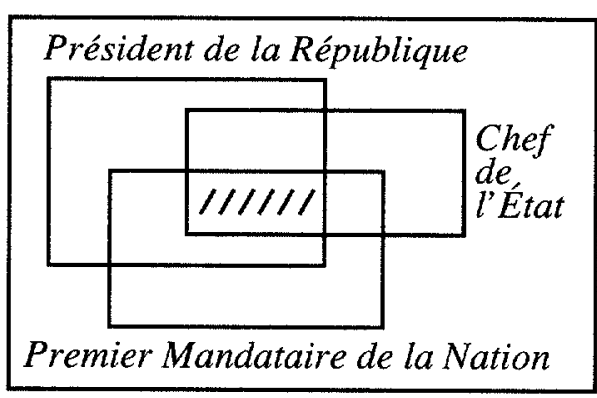

2. Parasynonymes

\section{2. Co-hyponymie}

Macroparadigme sémant.-synt.

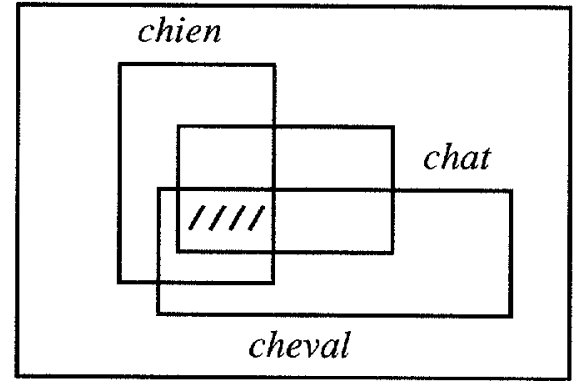

$\mathbf{X}$

Co-hyponymes

Structures : ensemble-intersection/ensemble différence

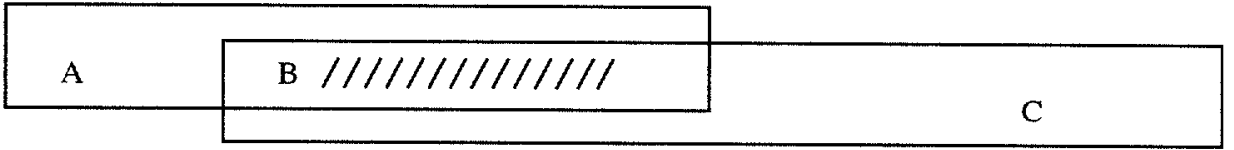

A

Paradigme de référentialité : même référence cognitive, références affectives distinctes.

Paradigme de distribution : commutables en de nombreux contextes sémantiques.

Ensemble-différence sémique: peu de sèmes, neutralisables selon le contexte et annu-

lables avec le temps.

Relation d'équivalence ou d'implication réciproque.

B

Rattachés à un terme surordonné.

Relation d'opposition transitive (ensemble-intersection sémique). Paradigme de la sémantique grammaticale: les deux ont la possibilité de recevoir l'incidence de signes grammaticaux compatibles avec la classe lexicale à laquelle ils appartiennent.

Paradigme syntagmatique : même possibilité de combinatoire syntagmatique.

$\mathrm{C}$

Paradigme de référentialité : références cognitives et affectives distinctes.

Paradigme de distribution: non commutables entre eux du point de vue sémantique.

Ensemble-différence sémique: sèmes non neutralisables (dans le cas de co-hyponymes distincts).

Impossibilité d'équivalence.

Considérons, à présent, les différents degrés de co-hyponymie: co-hyponymie proche et co-hyponymie distante. Comme nous l'avons signalé plus haut, Galisson (1973: 103) montre que les co-hyponymes se définissent comme des termes ayant un contenu très semblable, appartenant à un même microsystème, celui-ci défini par un hypéronyme commun à tous les hyponymes de l'ensemble. Cet auteur observe, également, que le degré de proximité entre ces contenus varie: certains possèdent un ensemble-intersection, un dénominateur sémantique commun ample: ce sont les co-hyponymes proches; d'autres ont un noyau sémique assez réduit par rapport au précédent: ce sont les co-hyponymes distants. Comme on peut le noter, le même type de relation entre les parasynonymes et les co-hyponymes lato sensu se vérifie entre les 
ensembles co-hyponymes proches et distants. Schématiquement, de telles relations peuvent être ainsi formalisées:

\section{Co-hyponymes proches}

A. parler

«émettre des sons vocaux»

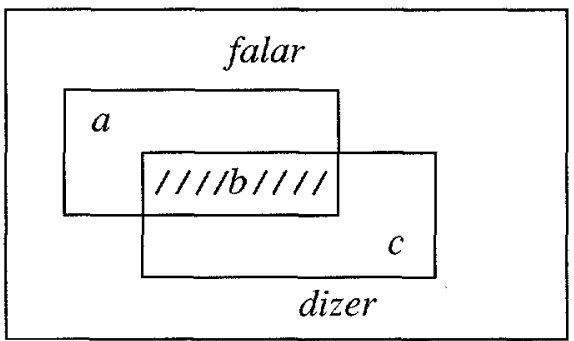

\section{Co-hyponymes distants \\ B. animal}

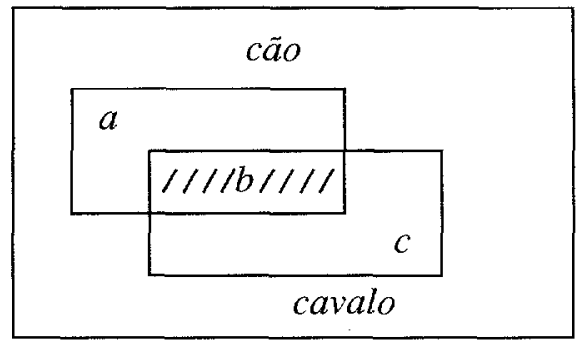

Les co-hyponymes des deux ensembles sont en relation d'opposition transitive, mais la proximité sémantique des éléments de l'ensemble $\mathbf{A}$ est beaucoup plus grande que celle des éléments de l'ensemble $\mathbf{B}$.

Sur l'axe des termes impliqués dans une relation sémantique, selon la formalisation donnée par Pottier (1968: 69), pour la question de «marque», les éléments se distribuent de la façon suivante:

Distinction proposée

Terme marqué $(+)$

Terme non-marqué (-)

Distinction non-proposée

Terme complexe ou générique $(0)$

Généralement, la co-hyponymie proche se vérifie dans un microsystème qui comprend deux co-hyponymes; l'un est le terme marqué $(+)$; l'autre, le terme non-marqué $(-)$, ce dernier étant la forme utilisée, également, pour exprimer la neutralisation (0) entre les deux premiers et, pour cela même, son hypéronyme. Dans le cas de l'ensemble parler/dire, par exemple, dire est le terme marqué, parler, le terme non-marqué, dans la mesure où ce dernier peut être employé soit dans son sens spécifique, soit comme terme extensif, comprenant, dans cette dernière condition, lui-même et l'élément marqué; ainsi employé, il assume la fonction d'hypéronyme du microsystème en question.

Le système linguistique de la langue portugaise, dans sa phase actuelle, en ce qui concerne son réseau lexico-sémantique et en considérant certaines synstraties et synphasies, tend à subir une recatégorisation d'ensembles comme falar/dizer et olhar/ver, etc.; de co-hyponymes distants, ils se transforment lentement et imperceptiblement en co-hyponymes proches. De cette manière, du point de vue du stade actuel du système de la langue portugaise, en considérant le réseau lexico-sémantique en synchronie, de tels ensembles sont constitués de co-hyponymes proches, dans des synstraties et des synphasies bien déterminées.

De cette façon, nous avons:

a) $«$ Falar sem dizer $\gg=(a+b)-c$

Cf. «Falou, falou e não disse nada».

b) $\langle$ Falar e dizer $\rangle=(\mathrm{a}+\mathrm{b})+\mathrm{c}$

Cf. «Falou e disse». 
c) $\langle$ Dizer» $=\mathrm{c} \supset(\mathrm{a}+\mathrm{b})$

Cf. «Disse coisas muito importantes».

d) $\ll$ Falar» (dans son emploi générique ou complexe) $=\mathrm{a} \cong(\mathrm{a}+\mathrm{b}+\mathrm{c})$

Cf. «Amanhã o Presidente falará à nação».

Une formalisation équivalente peut être faite dans l'analyse et la description de microsystèmes qui comprennent des paires comme escutarlouvir, olhar/ver, entender/compreender, entre autres.

Peuvent s'appliquer à tous ces exemples les réflexions présentées par Coseriu (1980: 76), à propos du processus de neutralisation sémantique et de l'extension de la notion de «marque», de l'analyse phonologique aux analyses morphologique, sémantique et syntaxique: le terme caractérisé ou «marqué», qui possède un caractère supplémentaire, en plus de la part commune, est le terme positif ou «intensif», parce qu'il est concentré sur une valeur déterminée, alors que l'autre terme sera considéré comme négatif ou «extensif» dans la mesure où il peut assumer les deux valeurs. De cette façon, le terme qui ne contient que la part sémantique commune peut aussi être appelé «neutre», puisqu'il n'implique pas nécessairement le contraire de l'autre terme.

Dans ces conditions, le terme marqué a une seule valeur de langue, tandis que le terme non marqué a deux valeurs, également au niveau de la langue, dont l'une est le contraire du terme marqué, et l'autre est le terme générique (qui comprend aussi le terme marqué). Ainsi, suivant l'exemple donné par Coseriu (1980:76), jour peut être jour comme le contraire de nuit, mais peut être aussi jour en tant que «jour + nuit». Dans sa conception, ce type de relations d'opposition «neutralisables» caractérise les langues en référence aux systèmes logiques et scientifiques, dans lesquels les oppositions sont univoques et ont uniquement la forme [A/non-A], c'est-à-dire que quelque chose qui est $\mathbf{A}$ ne peut pas être en même temps non-A, alors que les oppositions linguistiques ont presque toujours la forme:

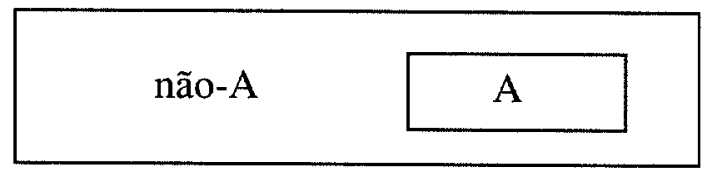

Autrement dit, une chose peut être non-A et aussi A (mais non le contraire: A ne peut être non-A).

Cela semble être le cas des microsystèmes cités plus haut, que nous considérons ici (nous l'avons déjà dit), comme un type possible de co-hyponymie proche.

Nous considérons, encore, comme des co-hyponymes proches des paires de termes qui appartiennent à un microsystème déterminé, rattachés à un même hypéronyme mais qui, contrairement à l'exemple examiné ci-dessus, ne sont pas caractérisés par l'opposition marqué/non-marqué et, de plus, ont comme hypéronyme un terme différent des deux hyponymes qu'il contient. Cela est le cas par exemple de termes comme émigration/immigration, qui possèdent dans le terme neutre, migration, l'archilexème de l'ensemble, son hypéronyme, qui neutralise dans des contextes déterminés l'opposition sémantique fonctionnelle qui existe entre les premiers et, du point de vue du signifié, comprend uniquement les traits communs aux deux termes. Cf. le cas d'«oiseau migrateur».

Nous pouvons inclure dans ce dernier cas l'ensemble anaphore/cataphore, qui, dans la conception de Maillard (1974: 55), peut voir son opposition neutralisée dans certains contextes, étant, alors, substitué par le terme diaphore, archilexème de ce microsystème qui transcende l'opposition «référence antérieure/référence postérieure» pour 
désigner la «référence contextuelle intralinguistique en général». Ainsi, donc, le terme diaphorique n'est pas vectoriellement orienté, il s'applique à des fragments d'énoncés qui établissent des références endodiscursives quelconques. Par conséquent, diaphorique s'oppose à aphorique, en constituant un ensemble de termes qui, dans l'arborescence de ce microsystème, précède la sous-classe des diaphoriques, anaphoriques et cataphoriques. En d'autres termes, dans une première opposition, nous dirons qu'un fragment énonciatif quelconque est soit «aphorique» soit «diaphorique», relativement au contexte: l'un est auto-incident, l'autre doté de référence contextuelle intralinguistique générique; dans une seconde opposition, on spécifie si le fragment énonciatif suppose l'énoncé précédent (diaphore anaphorique) ou s'il se réfère à l'énoncé (ou fragment) subséquent (diaphore cataphorique).

Il convient de signaler que, tant dans l'ensemble émigration/immigration que dans l'ensemble anaphoriquelcataphorique, en plus de la relation de co-hyponymie proche, observable entre le contenu des deux termes, on vérifie aussi, dans chaque ensemble, une relation de paronymie entre ces mêmes termes. Schématiquement, nous avons:
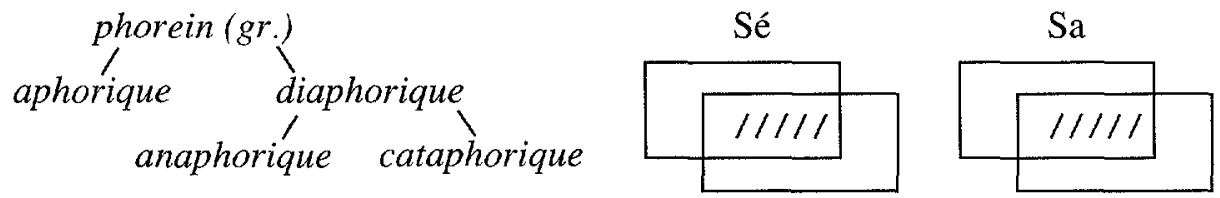

Considérons un dernier aspect, celui du mouvement de recatégorisation. Les microsystèmes examinés ci-dessus, comme tous les éléments et structures de langue, sont mutables à condition d'être observés en panchronie, dans la mesure où le système linguistique se soutient dans une tension dialectique conservation/mutation. La délimitation précise de tous ces ensembles, donc, n'est pas toujours possible, ne se présente pas de façon nette, car les différences et les ressemblances doivent être analysées et décrites à la lumière d'un temps, d'un lieu, d'une classe sociale, d'un univers de discours ou même d'un discours manifesté. Pour cette raison, certaines unités lexicales peuvent subir des déplacements significatifs dans le réseau lexico-sémantique et sémantico-syntaxique; des co-hyponymes distants peuvent devenir des co-hyponymes proches (cf. regarder/voir, par exemple, par emploi réitératif de haute fréquence, sans focalisation sémantique précise); des co-hyponymes proches peuvent se déplacer vers l'ensemble parasynonymique; des éléments de ce dernier peuvent en venir à intégrer l'ensemble synonymique; des mouvements inverses peuvent avoir lieu en différentes diachronies, diatopies, diastraties et diaphasies.

En effet, la fréquence d'actualisation d'unités lexicales, dans des discours déviatoires ou non, détermine leur permanence dans l'un ou l'autre des ensembles supracités. Il est nécessaire, arrivés à ce point, de souligner le fait qu'un discours peut coïncider avec une norme (degré zéro), ou rompre avec une norme (écart); un tel écart, dans la formalisation de Lopes (1976: 70), peut être interprété soit comme une erreur (écart dysphorique, non poétique), soit comme une intention (écart euphorique, poétique), en fonction du temps, du lieu, de la classe sociale ou de l'univers de discours - ce qui est euphorique, selon l'un de ces paramètres, peut être senti comme dysphorique, selon un autre paramètre. Ainsi, par exemple, regarder/voir, employés dans des discours non déviatoires ou dans des discours déviatoires euphoriques, s'intègrent aux microsystèmes co-hyponymiques distants ou proches $(\mathrm{a} \quad \mathrm{b} 0)$; employés dans des discours déviatoires dysphoriques, ils sont soit placés dans une relation d'équivalence $(a \sim b)$, distribués dans 
le microsystème des parasynonymes, soit placés dans une relation d'identité $(a=b)$, insérés dans le microsystème des synonymes.

Toutefois, le passage de l'un à l'autre ensemble, étape finale du processus de recatégorisation, ne se fait pas, lorsque l'écart est constaté dans un seul discours manifesté, ou dans les premiers discours manifestés où il apparaît. Nous avons ici seulement l'éventuel début d'un processus de recatégorisation. Dans cette phase initiale, l'emploi déviatoire, euphorique ou dysphorique, se caractérise par sa nature néologique sémantique, par son fort taux d'information, qui provoquent l'impact de la nouveauté lexicale et le sentiment de néologicité. En effet, au moment de sa création, l'écart provoque un effet de sens appréciable, si l'on considère, comme Genouvrier et Peytard (1974: 403), que l'effet de style est détectable par la surprise qu'il provoque chez le lecteur/auditeur. Cependant, la répétition des actualisations de l'écart, l'augmentation de sa fréquence tendent à provoquer une réduction du taux d'information, une diminution de la conscience de néologicité, la disparition de l'impact de la nouveauté lexicale (Barbosa 1989: 153), la progressive annulation de l'effet de style provoqué par cet écart. À ce propos, Marcellesi (1974: 96) essaie de montrer que l'importance du contenu de l'information véhiculée par les mots, c'est-à-dire leur charge d'information, est inversement proportionnelle à la fréquence de leur utilisation. Dans cette même ligne de pensée, Genouvrier et Peytard (1974 : 403), en citant Martinet d'ailleurs, affirment qu'«on peut attribuer l'effet de style au degré de probabilité des unités linguistiques dans un contexte donné» (Martinet); plus le degré de probabilité est élevé, plus l'effet de style est léger; plus le degré de probabilité est faible, plus l'effet est vif. Comme l'information d'une unité est inversement proportionnelle à sa probabilité, le style se définit comme une recherche systématique de la «densité informationnelle»: ce sont les mots rares, c'est-à-dire les plus inattendus, et donc ceux qui s'éloignent le plus de la norme, qui apportent le plus d'information. De cette façon, à mesure que de tels écarts voient leur fréquence augmenter, ils perdent progressivement leur caractère de néologicité, de tels vocables en viennent à intégrer une norme, et ainsi se conclut, alors, le processus de recatégorisation.

Les mouvements de recatégorisation peuvent être ainsi schématisés :

a) synonymes

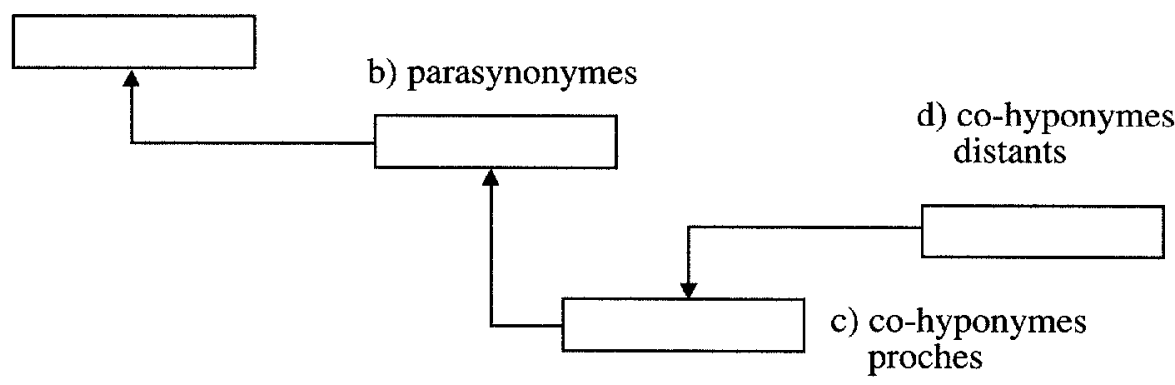

Cette dynamique provient de la nature même du système linguistique, qui, comme nous l'avons signalé plus haut, se maintient dans une tension dialectique - indispensable à son fonctionnement - entre deux forces contraires, non incompatibles mais complémentaires, celle de conservation et celle de mutation (Pais 1979: 103-123). Cet équilibre dynamique et cette tension sont observables dans n'importe quelle étape synchroniquement considérée d'une langue, sous trois aspects: la conservation d'une 
grande part du lexique, l'apparition de nouvelles unités lexicales, la disparition de certaines autres (Barbosa 1989: 132). Cette oscillation a lieu sans que les locuteurs se rendent compte que la langue n'est plus égale à elle-même, alors qu'ils parlent.

Devant ce que nous venons de voir, dans l'élaboration du système de renvois de l'œuvre lexicographique, particulièrement dans le dictionnaire de langue, il est souhaitable que de telles questions soient prises en compte. Ainsi, par exemple, il convient de distinguer les co-hyponymes distants des formes équivalentes et de ne pas appliquer aux premiers le renvoi voir. Au contraire, ce type de renvoi, tout comme le renvoi $D q$ (descripteur équivalent) conviennent aux parasynonymes. Dans le cas des vocabulaires technico-scientifiques, il faut ajouter, aux exigences précédentes, le problème de la synonymie qui s'établit entre un terme technique et son correspondant vulgaire, ce qui oblige à faire deux renvois, $D q$ ou $F E$ (forme équivalente). Enfin, lorsqu'il s'agit d'un glossaire d'une cuuvre déterminée, il est nécessaire de prendre en compte, également, l'équivalence contextuelle de formes lexicales, la question de la synonymie devenant, alors, plus emphatique.

\section{RÉFÉRENCES}

BARBOSA, M. A. (1989): «Reflexões sobre o projeto lexicográfico : análise e descrição da forma do conteúdo da unidade lexical», Estudos lingüísticos XVIII, Anais de Seminários do GEL, pp. 65-75.

BARBOSA, M. A. (1989): «Co-hiponímia e parassinonímia : a tênue fronteira entre relações semióticas e suas implicações lexicográficas», 42a Reuniāo Anual, SBPC, Anais (Comunicaçôes), Ciência e Cultura, 42-7, pp. 325-326.

BARBOSA, M. A. (1989): Léxico, produção e criatividade. Processos do neologismo, $2^{\mathrm{e}}$ édition, São Paulo, Global.

BRECKLE, Herbert E. (1974) : Sémantique, Paris, Armand Colin.

COSERIU, E. (1980) : Lições de lingüística geral, Rio de Janeiro, Ao livro técnico.

GALISSON, R. (1973): «Pour une méthodologie de l'enseignement du sens étranger», Études de linguistique appliquée, 11, pp. 87-126.

GECKELER, H. (1976) : Semántica estrutural y teoría del campo léxico, Madrid, Gredos.

GENOUVRIER, E. et J. PEYTARD (1974): Lingüística e ensino do Português, Coimbra, Almedine.

LOPES, E. (1976) : Fundamentos da lingüística contemporânea, São Paulo, Cultrix.

LYONS, J. (1979): Introduçäo à lingüística teórica, São Paulo, Nacional/EDUSP.

MAILLARD, M. (1974): «Essais de typologie des substituts diaphoriques», Langue française, 21, pp. 55-71.

MARCELLESI, C. (1974): «Néologie et fonctions du langage», Langage, 36, pp. 95-102.

MULLER, C. (1968): Initiation à la statistique linguistique, Paris, Larousse.

PAIS, C. T. (1979): «Les tensions et les parcours de production du processus sémiotique», Acta Semiotica et Linguistica, 3, pp. 103-123.

POTTIER, B. (1968): Presentación de la lingüística, Madrid, Alcalá.

REY, A. et S. DELESALLE (1979) : «Problèmes et conflits lexicographiques», Langue française, 43, pp. 4-26. 